\title{
Differential RF MEMS interwoven capacitor immune to residual stress warping
}

\section{A.M. Elshurafa and K.N. Salama}

Electrical Engineering, Physical Sciences and Engineering Division, King Abduallah University of Science and Technology (KAUST), Thuwal, Saudi Arabia, 23955-6900.

Abstract: An RF MEMS capacitor with an interwoven structure is designed, fabricated in the PolyMUMPS process, and tested in an effort to address fabrication challenges usually faced in MEMS processes. The interwoven structure was found to offer several advantages over the typical MEMS parallel-plate design including eliminating the warping caused by residual stress, eliminating the need for etching holes, suppressing stiction, reducing parasitics, and providing differential capability. The quality factor of the proposed capacitor was higher than 5 throughout a $2 \mathrm{GHz}-$ $10 \mathrm{GHz}$ range and the resonant frequency was in excess of $20 \mathrm{GHz}$.

\section{Introduction}

Radio frequency microelectromechanical systems (RF MEMS) technologies have received significant research attention in the past 15 years in an effort to replace bulky off-chip components [1-3]. With respect to RF fixed capacitors specifically, the majority of capacitors used in MEMS or CMOS are of the parallel-plate type. A different type of fixed capacitors known as fractal capacitors was introduced in CMOS [4] for capacitance density enhancement.

Several process-related considerations specifically applicable to MEMS capacitors are not found in their CMOS counterparts. For example, capacitors in CMOS are not eventually released. However, because MEMS usually contain moving parts, the sacrificial layer that resides between the MEMS capacitor plates is etched 
away; etching/drying can cause stiction. Further, whenever etching is to be performed, etching holes are required [5]. Other problems that parallel plate capacitors (PPC) suffer from include the warping caused by the residual stress especially for larger plates [5].

In this paper, we use the interwoven structure specifically and create the first MEMS interwoven capacitor (IWC). The interwoven structure was inspired from its ubiquity; it is found in baskets, hair braids, bamboo sofas, carpets and rungs made with looms, etc; it is also a natural continuation to the previously reported interleaved and woven structures $[4,6]$. Using the interwoven structure, several advantages become readily attainable without any fabrication intervention or additional postprocessing as will be shown.

\section{Proposed capacitor structure}

A 3D isometric view of the proposed IWC, as generated by SEMulator3D (www.coventor.com), is shown in Fig. 1 in which the overall structure including the measurement pads are depicted; a close up image is also provided and explains the capacitances present within the structure. Further, a microphotograph of the fabricated capacitor is shown in Fig. 2 along with a close up scanning electron microscope (SEM) image. The interwoven capacitor is created and connected to the measurement pads in such a way that any two adjacent depositions possess opposite polarities to attain horizontal capacitances. Moreoever, any two depositions that are on top of each other are also of opposite polarities to attain vertical capacitances. Fig. 1 explains these capacitances visually in detail.

We choose to build the MEMS IWC in the PolyMUMPS [7] process for two important reasons. First, the PolyMUMPS process allows easy and effective 
connections between the top and bottom layers using vias. Secondly, and once the weave is created, the complete structure can be suspended a considerable distance above the substrate (i.e. 2.6um), which reduces parasitic coupling [8]. The resonant frequency, $f_{R}$, of a capacitor is generally given by:

$$
f_{R}=\frac{1}{2 \pi \sqrt{L C_{t}}}
$$

where $L$ is the inherent inductance present due to the connections and the lines within the structure itself, and $C_{t}$ is the total capacitance which comprises the capacitance from the structure itself $(C)$ and the parasitic capacitance $\left(C_{p}\right)$, i.e. $C_{t}=C+C_{p}$. The parasitic capacitance can be computed using: $C_{p}=\varepsilon A / d$, where $\varepsilon$ is the dielectric constant, $A$ is the area of the plate, and $d$ is the distance separating the plate from the substrate. Because the area of the plate is small (not a typical rectangular shape) and because the structure is far from the substrate, $C_{p}$ decreases and in turn $f_{R}$ increases as (1) suggests.

One disadvantage of the IWC is that it possesses many vias, which are required to connect the top and bottom layers together. These vias cause a decrease in the quality factor $(Q)$.

\section{Simulation}

The finite element software COMSOL (www.comsol.com) was used to simulate the capacitance of the IWC because a closed-form expression is difficult to obtain given the complicated fringing fields present. Due to symmetry, it is possible to simulate a small part of the capacitor only (Fig. 3); this reduces the time and memory requirements of computation significantly. The capacitance extracted for the structure in figure $3 \mathrm{a}$ was $0.0156 \mathrm{pF}$; this translates to a total capacitance of approximately 
$0.4 \mathrm{pF}$ for the complete structure without the measurements pads and to ultimately $0.67 \mathrm{pF}$ including the measurement pads $[9,10]$.

\section{Advantages of the interwoven structure}

\subsection{Elimination of warping caused by residual stress}

MEMS structures generally suffer from warping due to residual stresses. Further, the residual stress itself varies across the wafer and from one run to another within the same process $[11,12]$. Thermal annealing is usually performed to suppress this warping.

With respect to the IWC, and because it comprises several small segments, the warping that will take place will be virtually nonexistent even if the dimensions of the plate becomes bigger. In evidence of that, we fabricated a large MEMS IWC with a side length of $330 \mu \mathrm{m}$ (bigger than the one shown in Fig. 2 which has a side length of $250 \mu$ ), and also fabricate a typical parallel plate capacitor (PPC) possessing the same dimensions (i.e. $330 \mu \mathrm{m}$ ); optical profiler images of these capacitors are shown in Fig. 4. As can be seen, very little warping is present in the MEMS interwoven capacitor despite being anchored at the sides only. However, the PPC suffered a considerable warping distance of $1.7 \mu \mathrm{m}$. Even if the dimensions of the plate become bigger than the above cases, the interwoven structure would still be immune to residual stress warping as long as the main segments creating the overall structure are small. Explicitly, the interwoven structure is more repeatable process-wise. For comprehensiveness, prolife analysis was also performed on the smaller interwoven capacitor (Fig. 2) and on a basic PPC of the same size. Once again, the warping in the IWC was not present while a bending distance of $1.2 \mu \mathrm{m}$ was present in the PPC case (results however are not included herein interest of brevity). 


\subsection{Eliminating the need of etching holes}

Because MEMS usually contain moving actuators, the sacrificial layer in fabrication processes is etched away eventually. In order to reduce the etching time required, etching holes are distributed within large structures $[5,13]$. By taking a close look at Figs. 1 and 2, it is easily observed that there is no need for etching holes and no need to optimize their number, size, or perforation configuration. The etchant will easily penetrate through the structure and etch the oxide lying underneath.

\subsection{Reducing stiction effects}

It is well known that long beams and bigger plates are more prone to experience stiction [14, 15]. The MEMS IWC, however, comprises lower small segments that are connected to other higher small segments, interchangeably, in a weave-like fashion. Thus, the stiction phenomenon no longer becomes a worrisome factor during fabrication.

\subsection{Reducing parasitic capacitances}

This benefit is especially important for RF applications. Because the IWC does not have a real typical bottom square/rectangular plate as usually the case is, the coupling capacitances that are created with the substrate are significantly reduced. If the structure were to be also created as high as possible from the substrate, a further reduction in the parastitics would occur. Consequently, the SRF and $Q$ should theoretically increase as explained in section 2, and measurement results that will follow support this hypothesis. This advantage is vital if RF MEMS are to genuinely replace bulky off-chip components. 


\subsection{Differential advantage}

In typical PPCs, the parasitic capacitance is mostly present between the bottom plate and the substrate [5-8]. Hence, one side will experience more parasitics when compared to the other side, which makes the capacitor not symmetrically differential (i.e. not balanced). Moreover, if one of the plates is made out of metal and the other is made of polysilicon (which is usually the case in MEMS), another situation of non-differentiality occurs because the resistivity of the metal plate is lower than that of polysilicon as detailed in [16]. One solution to these latter problems could be to split the capacitor into two, parallel, half-capacitors. In the case of the IWC, however, we can deduce by inspection that the parasitics are already divided almost equally between the two terminals and that the resistivity in both terminals are already the same making it fully symmetrical. Note also that this benefit is not restricted to MEMS only. To verify that, measurements were performed using an LCR meter from Agilent (www.agilent.com). Results have shown that the difference in the capacitance magnitude between measurements when the probes are interchanged is less than $5 \mathrm{fF}$ for the IWC. Table 1 summarizes these benefits and compares it to the typical PP case.

Table 1. Summary of the benefits acquired from using an interwoven structure versus a parallel plate structure.

\begin{tabular}{|c|c|c|}
\hline Criterion & Typical MEMS PPC & MEMS IWC \\
\hline Warping due to residual stress & Present & Not present \\
\hline Etching Holes & Required & Lenger required \\
\hline Stiction & More likely to occur & $\begin{array}{c}\text { Suppressed } \text { - enhances } Q \\
\text { and/or delays its drop until } \\
\text { higher frequencies are reached }\end{array}$ \\
\hline $\begin{array}{c}\text { Parasitic Capacitance } \\
\text { Cymmetric Differential } \\
\text { Capability (i.e. substrate } \\
\text { parasitics) }\end{array}$ & $\begin{array}{c}\text { Significant - reduces } Q \text { and/or } \\
\text { quickly }\end{array}$ & Fully differential \\
\hline
\end{tabular}




\section{Measurements and comparison}

Fig. 5 shows $S_{11}$ measurements for the basic IWC from $2 \mathrm{GHz}$ to $10 \mathrm{GHz}$, where the capacitance and $Q$ for the complete band are summarized in Fig. 5a and a sample response measurement is shown in Fig. 5b. The maximum $Q$ is 10 at $5 \mathrm{GHz}$ and $Q$ was above 5 throughout the $2 \mathrm{GHz}-10 \mathrm{GHz}$ range because the structure was suspended 2.6 $\mu \mathrm{m}$ above the substrate and hence reduced parasitics. Further, simulation and measurement results exhibit good agreement.

For comparison and reliability purposes, we provide in Fig. 6a measurement results for six IWCs and one PPC from $1 \mathrm{GHz}$ to $3 \mathrm{GHz}$. As can be seen, the performance of the six IWCs is nearly identical and we prove that they are indeed reliable with no mechanical factors considered worrisome. Moreover, it is apparent from Fig. 6a that the SRF of the IWC is much higher than that of the PPC.

One final measurement was performed on a range from $15 \mathrm{GHz}$ to $20 \mathrm{GHz}$ as shown in Fig. $6 \mathrm{~b}$ in an effort to detect the SRF of the device. It is clear how the SRF is higher than 20GHz. However, the high SRF was obtained at the cost of a smaller capacitance value. No further measurements at higher frequency ranges were performed because a one-port measurement at such frequencies will not yield accurate results. We compare in Table 2 the SRFs and $Q$ s of previously reported capacitors in PolyMUMPS with the interwoven capacitor presented herein.

Table 2. Comparative study

\begin{tabular}{|c|c|c|c|c|c|c|}
\hline Criterion & $\begin{array}{c}\text { Woven } \\
{[6]}\end{array}$ & $\begin{array}{c}\text { Interleaved } \\
{[6]}\end{array}$ & $\begin{array}{c}\text { PPC } \\
{[9]}\end{array}$ & $\begin{array}{c}\text { PPC } \\
{[10]}\end{array}$ & $\begin{array}{c}\text { PPC } \\
{[17]}\end{array}$ & This work \\
\hline $\boldsymbol{C}(\mathbf{p F})^{\boldsymbol{\bullet}}$ & 1.1 & 1.2 & 1.9 & 1.4 & 2 & 0.7 \\
\hline $\boldsymbol{Q}$ & 6 at $2 \mathrm{GHz}$ & 3.5 at $2 \mathrm{GHz}$ & 16 at $1 \mathrm{GHz}$ & 14 at $2 \mathrm{GHz}$ & 12 at $2 \mathrm{GHz}$ & $\begin{array}{c}10 \text { at } 5 \mathrm{GHz} \\
5 \text { at } 10 \mathrm{GHz}\end{array}$ \\
\hline SRF (GHz) & 10 & 10 & 11 & $<8^{\boldsymbol{\vartheta}}$ & $<8^{\boldsymbol{\nu}}$ & $>20$ \\
\hline
\end{tabular}

* Measured at $1 \mathrm{GHz}$

Estimated from measurements

As can be seen, the SRF reported here is significantly higher than the rest of the 
capacitors. Further, previous papers report the $Q$ s at $1 \mathrm{GHz}$ or $2 \mathrm{GHz}$ because the $Q$ deteriorates rather quickly beyond this range. The IWC, on the other hand, can provide an acceptable $Q$ up to $10 \mathrm{GHz}$. It is worth mentioning also that $Q$ did not reach higher than 10 even at the $2 \mathrm{GHz}$ due to the many vias present in the structure. Finally, we note that the capacitors included in Table 2 are all two-layer capacitors rather than single-layer capacitors $[6,18]$ to ensure a fair comparison since the IWC is a two-layer capacitor.

\section{Conclusion}

We have shown that the IWC in MEMS offers several desirable characteristics over the conventional PPC. Among the benefits attained were the high SRFs, the differential capability, and immunity to residual stress warping. All these benefits were acquired without any fabrication intervention or post-processing. Further, the reliability of these capacitors was also examined. Finally, these advantages were acquired at the expense of a lower capacitance value when compared to the conventional PPC.

\section{Acknowledgments}

The authors would like to thank Mr. X. Yu, Dr. M Hedhili, Dr. A. Behzad, and Dr. C.

Zhao at the Advanced Nanofabrication, Imaging, and Characterization Core Laboratories in King Abdullah University of Science and Technology (KAUST) for assistance in measurements and imaging.

\section{References}

[1] El Gmati I., et al.: 'Variable RF MEMS fluidic inductor incorporating lamination process', IET Micro \& Nano Letters, 2010, 5, pp. 370-373. 
[2] Ghodsian B., Bogdanoff P., Hyman D.: 'Wideband DC-contact MEMS series switch', IET Micro \& Nano Letters, 2008, 3, pp. 66-69.

[3] Li L., Uttamchandani D.: 'MEMS microwave device with switchable capacitive and inductive states', IET Micro \& Nano Letters, 2008, 3, pp. 77-81.

[4] Samavati H., Hajimiri A., Shahani A., Nasserbakht G., Lee T.: 'Fractal capacitors', IEEE J. Solid-State Circuits, 1998, 33, pp. 2035-2041.

[5] Elshurafa A.M., El-Masry E.I.: 'Design considerations in MEMS parallel plate variable capacitors', IEEE Midwest Symp. Circ. Syst., 2007, pp. 1173-1176.

[6] Elshurafa A.M., Salama K.N.: 'Two-layer RF MEMS fractal capacitors in PolyMUMPS for S-band applications', IET Micro \& Nano Letters, 2012, 7, pp. 419-421.

[7] Carter J., et al.: 'PolyMUMPS Design Handbook - Rev. 11', MEMSCAP Inc., Bernin, France.

[8] Elshurafa A.M., El-Masry E.I.: 'MEMS variable capacitance devices utilizing the substrate: I. Novel devices with a customizable tuning range', $J$. Micromech Microeng., 2010, 20, article id 045027.

[9] Bakri-Kassem M., Mansour R.R.: 'A high-tuning-range MEMS variable capacitor using carrier beams', Canadian J. Electr. Comp. Eng., 2006, 31, pp. 89-95.

[10] Dec A., Suyama K.: 'Microwave MEMS-based voltage-controlled oscillators', IEEE Trans. Microw. Theory Tech., 2000, 48, pp. 1943-1949.

[11] Al-Dahleh R., Mansour R.R.: 'High-Capacitance-Ratio Warped-Beam Capacitive MEMS Switch Designs', IEEE J. Microelectromech. Syst., 2010, 19, pp. 538-547.

[12] Elshurafa A.M., El-Masry E.I,: 'The effects of non-uniform nanoscale deflections on capacitance in RF MEMS parallel-plate variable capacitors', $J$. Micromech. Microeng., 2008, 18, article id 045012.

[13] Elshurafa A.M., El-Masry E.I.: 'Effects of etching holes on capacitance and tuning range in MEMS parallel plate variable capacitors', IEEE Int'l Workshop Syst. on Chip, 2006, pp. 221-224.

[14] Liu C.: Foundations of MEMS, Prentice Hall, 2006.

[15] Ranganathan R., Sivakumar G., Gale R., Dallas T.: 'Characterization of Stiction Accrual in a MEMS', IEEE J. Microelectromech. Syst., 2009, 18, pp. 1149-1159.

[16] Ionis G., Dec A., Suyama K.: 'Differential multi-finger MEMS tunable capacitors for RF integrated circuits', IEEE Int'l Microw. Symp., 2002, pp. 345-348.

[17] Dec A., Suyama K.: 'RF micromachined varactors with wide tuning range', IEEE Radio Freq. Integ. Cir. Symp., 1998, pp. 309-312.

[18] Elshurafa A.M., Radwan A.G., Emira A., Salama K.N.: 'RF MEMS Fractal Capacitors With High Self-Resonant Frequencies', IEEE J. Microelectrom. Syst., 21, pp. 10-12. 


\section{Figure Captions}

Fig. 1. A conceptual 3D isometric view of the interwoven capacitor, and an exaggerated view to show the details. The via connects the top layer (or plate) to the bottom one and the Anchor fixes the complete structure to the substrate. The capacitances present are: lateral capacitance between the top layers alone, lateral capacitance between the bottom layer alone, vertical capacitance between the top and bottom layers, and fringing capacitances throughout the capacitor.

Fig. 2. A microphotograph of the fabricated capacitor (left) and a close-up scanning electron microscope image (right).

Fig. 3. Simulation results from COMSOL for the capacitance extraction.

$a$ The structure that was created to extract the capacitance

$b$ Electrostatic simulation showing a surface plot for the shape of the electric filed above the capacitor

$c$ An arrow plot showing the complexity of the fringing fields' directions. The color scale bars in $b$ and $c$ represent the normalized electric potential.

Fig. 4. 3D oblique optical profile plots of both capacitors showing clearly residual stress bending in the PPC case.

$a$ The IWC.

$b$ The PPC.

Fig. 5. Measurement results of the MEMS IWC.

$a$ The capacitance and $Q$.

$b$ Sample measurement at $5 \mathrm{GHz}$ showing $Q>10$.

Fig. 6. Repeatability measurements and SRF detection.

a $S_{11}$ measurement for six interwoven capacitors and a basic PP capacitor.

$b$ Measurement showing that SRF is beyond $20 \mathrm{GHz}$. 


\section{Figure 1}

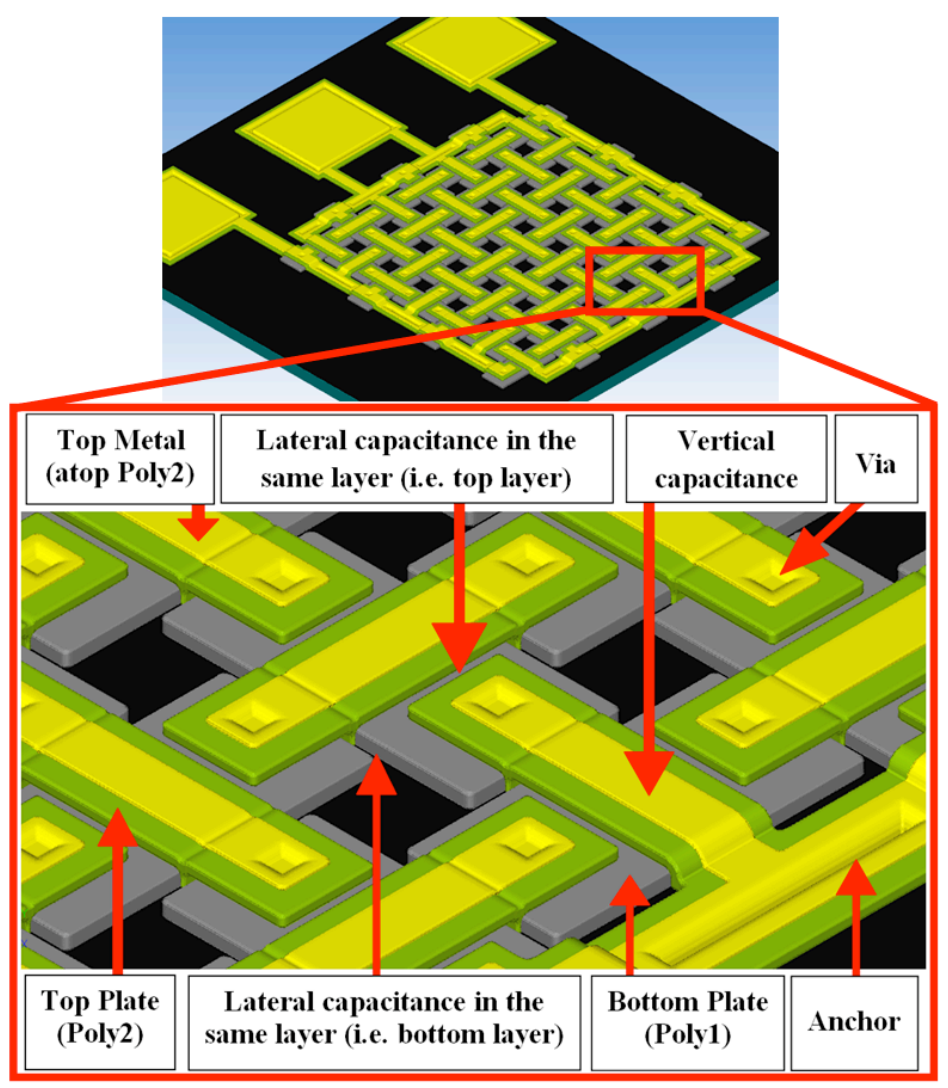

Fig. 1. A conceptual 3D isometric view of the interwoven capacitor, and an exaggerated view to show the details. The via connects the top layer (or plate) to the bottom one and the Anchor fixes the complete structure to the substrate. The capacitances present are: lateral capacitance between the top layers alone, lateral capacitance between the bottom layer alone, vertical capacitance between the top and bottom layers, and fringing capacitances throughout the capacitor. 


\section{Figure 2}

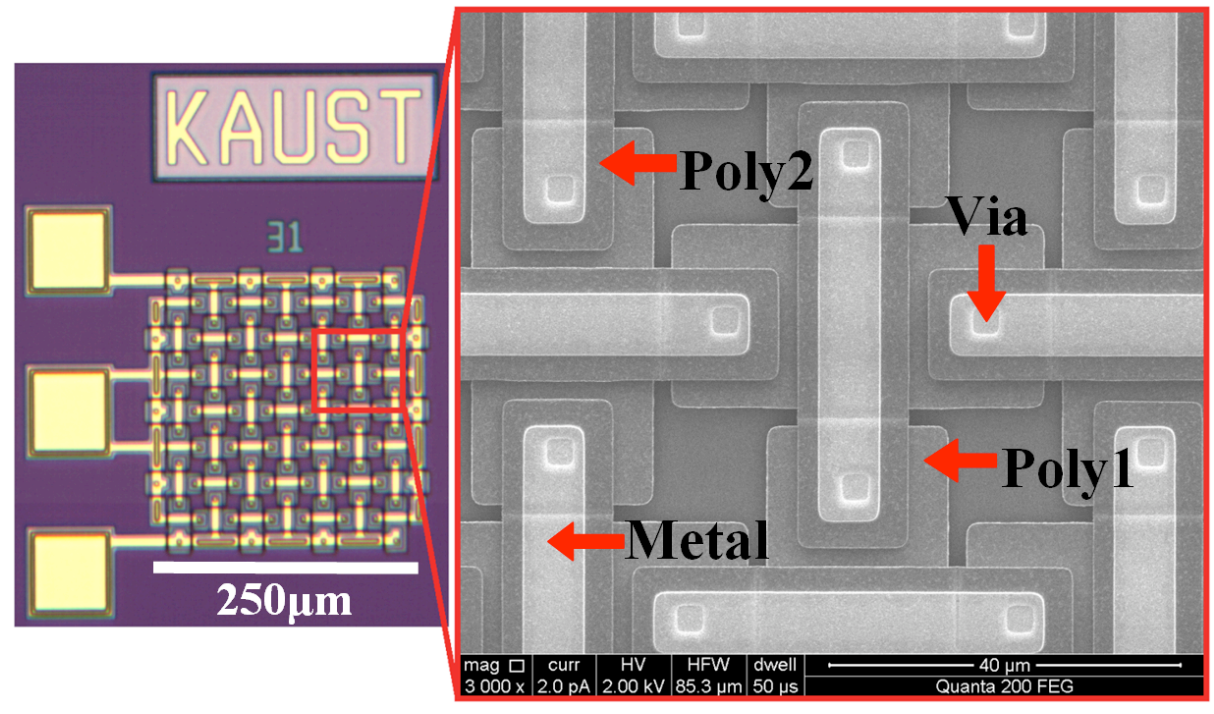

Fig. 2. A microphotograph of the fabricated capacitor (left) and a close-up scanning electron microscope image (right). 


\section{Figure 3}
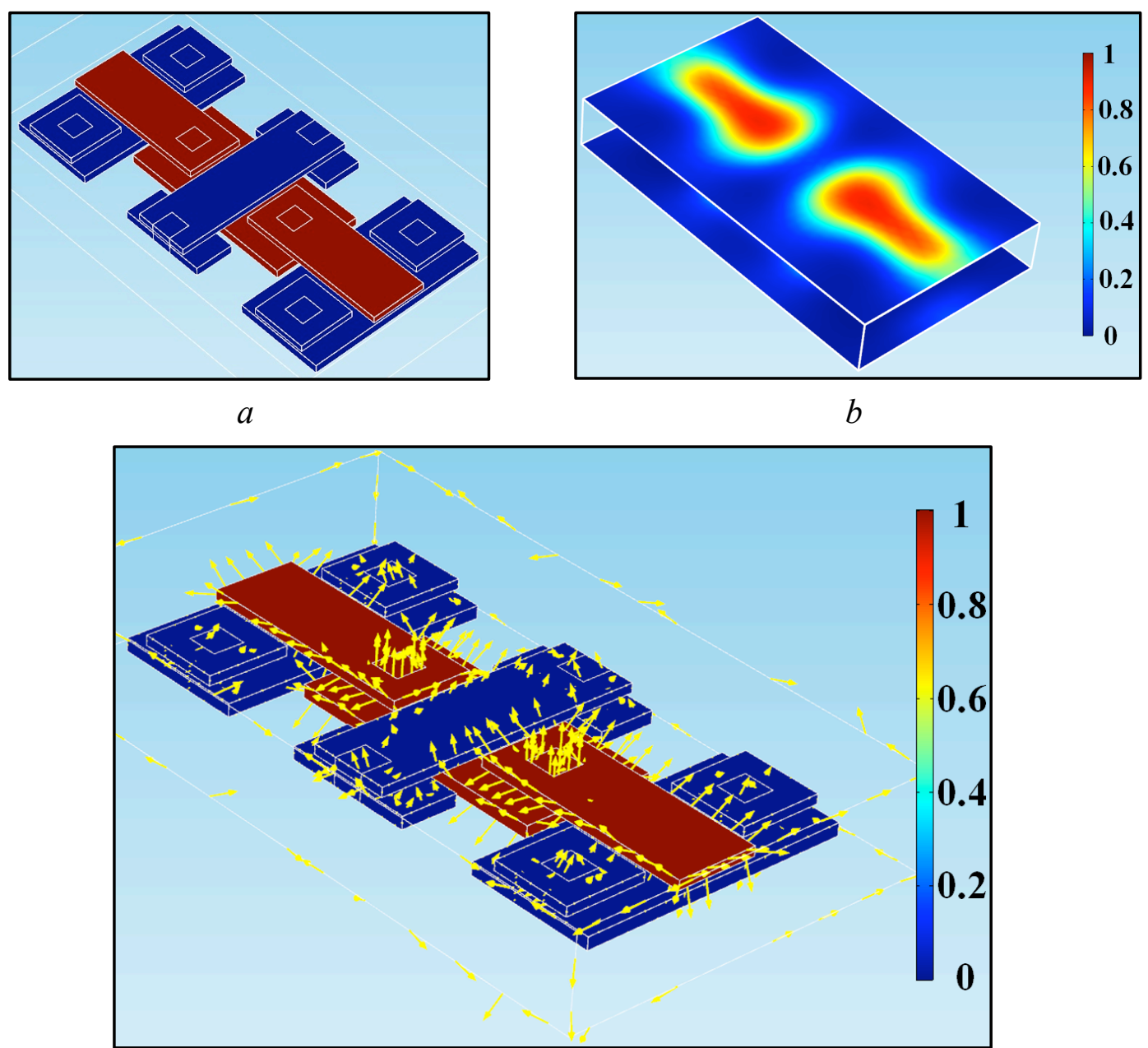

$c$

Fig. 3. Simulation results from COMSOL for the capacitance extraction. $a$ The structure that was created to extract the capacitance

$b$ Electrostatic simulation showing a surface plot for the shape of the electric filed above the capacitor

$c$ An arrow plot showing the complexity of the fringing fields' directions. The color scale bars in $b$ and $c$ represent the normalized electric potential. 


\section{Figure 4}
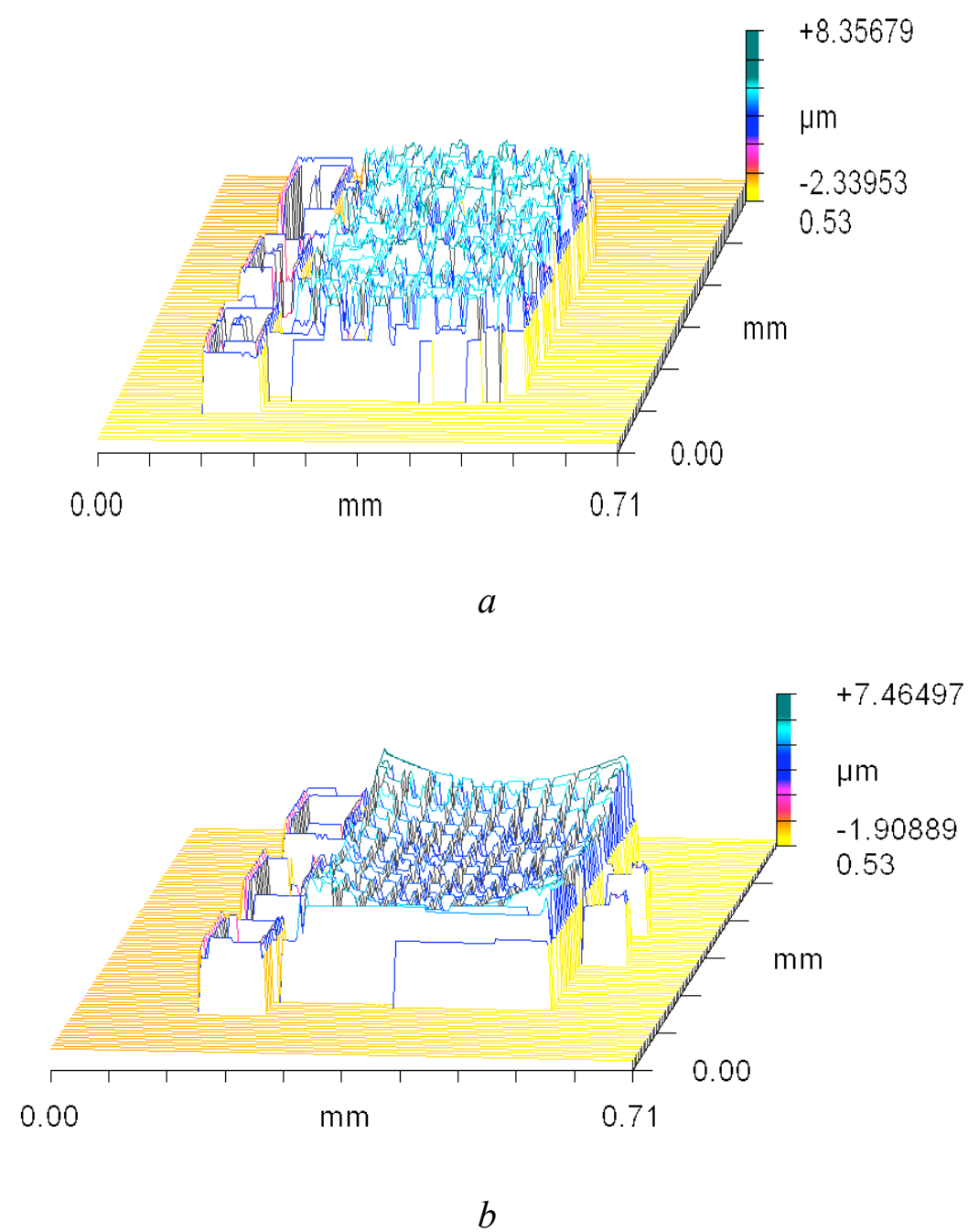

Fig. 4. 3D oblique optical profile plots of both capacitors showing clearly residual stress bending in the PPC case.

$a$ The IWC.

$b$ The PPC. 
Figure 5
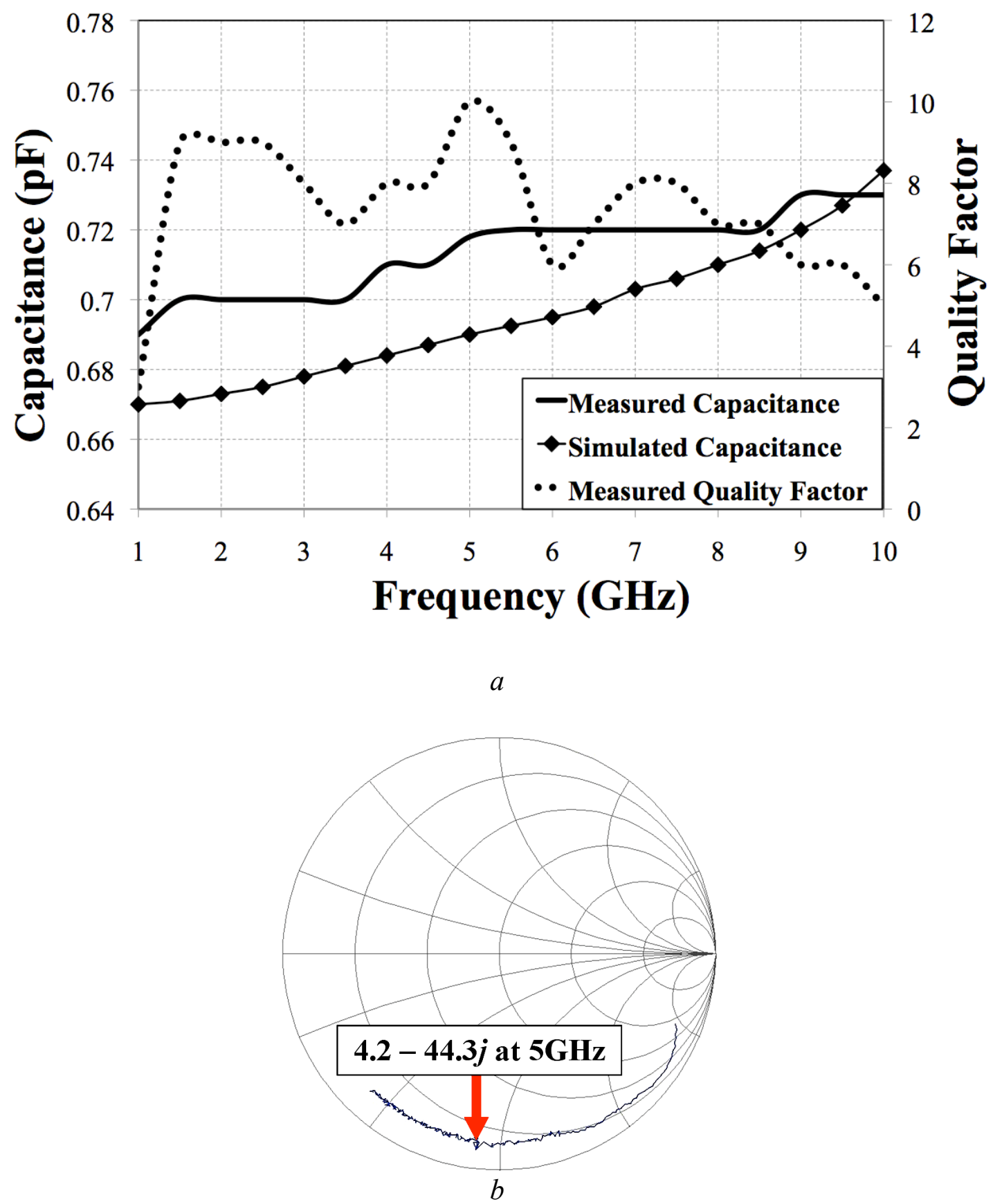

Fig. 5. Measurement results of the MEMS IWC. $a$ The capacitance and $Q$.

$b$ Sample measurement at $5 \mathrm{GHz}$ showing $Q>10$. 


\section{Figure 6}

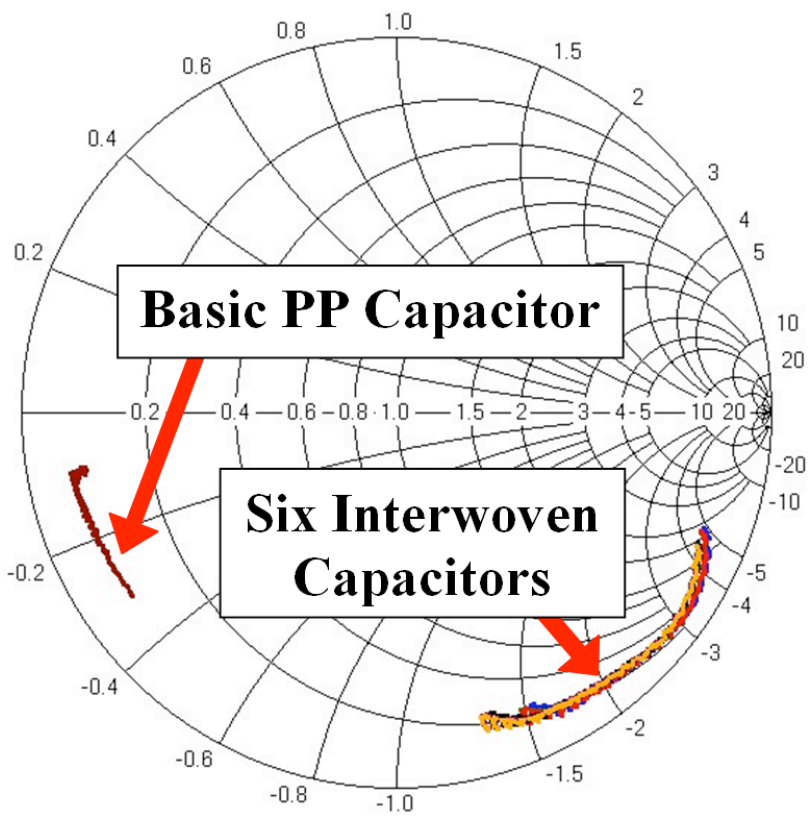

$a$

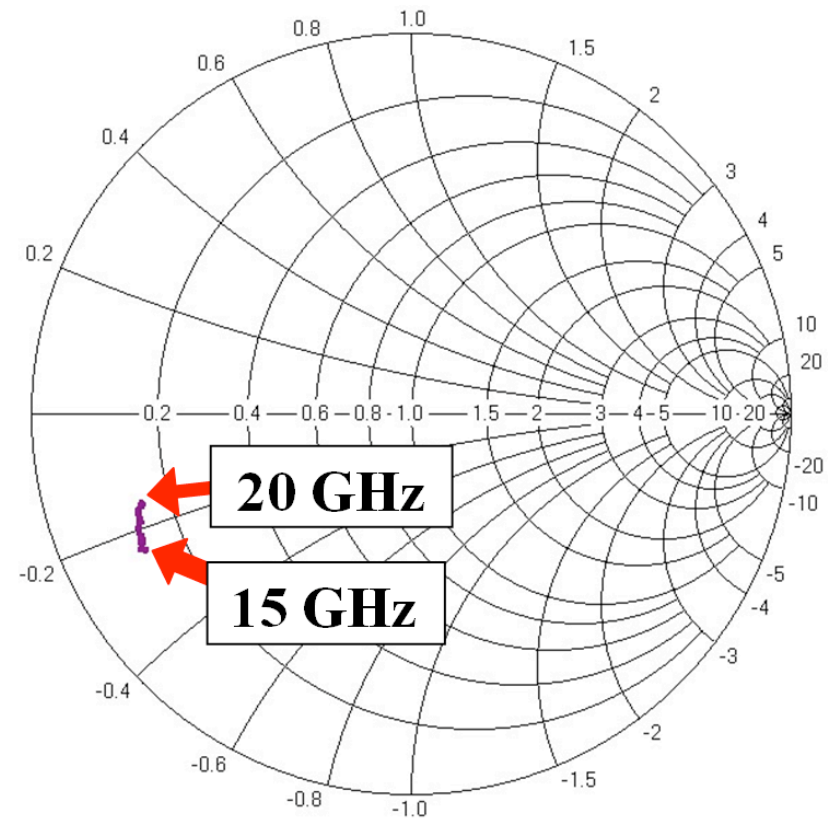

$b$

Fig. 6. Repeatability measurements and SRF detection.

a $S_{11}$ measurement for six interwoven capacitors and a basic PP capacitor. $b$ Measurement showing that SRF is beyond $20 \mathrm{GHz}$. 